\title{
PROTOPLASMA - Proton-driven plasma-wakefield experiment at Fermilab: Stages and Approach
}

\author{
J. C. T. Thangaraj ${ }^{*}$, C. S. Park*, J. D. Lewis*, P. Spentzouris*, W. An ${ }^{\dagger}$, W. Mori ${ }^{\dagger}$ and \\ C. Joshi ${ }^{\dagger}$ \\ *Fermi National Accelerator Laboratory, IL, USA \\ ${ }^{\dagger}$ Univ. of California, Los Angeles, CA, USA
}

\begin{abstract}
Generation of TeV-scale electron beams using conventional RF technology appears expensive for building the next generation of colliders. Proton-driven plasma-wakefield acceleration of electrons promises an alternative route to generate TeV-scale electron beams using existing proton machines. PROTOPLASMA is the proposed R\&D project at Fermilab that plans to use a proton beam driven plasma-wakefield to accelerate electrons. The project is planned in stages with the project's path guided by simulations. First, a $60-120 \mathrm{GeV}$ proton beam will be injected into 1-2 meters of plasma to observe selfmodulation instability in the proton beam. Next, an injected $5 \mathrm{MeV}$ electron beam will be accelerated by the plasma. In this paper, we report on the basic project plan and outline our staged approach. We report on first simulation results that show self-modulation of a proton bunch and discuss beam optics requirements and other limits.
\end{abstract}

Keywords: proton-driven accelerator, plasma wakefield, self-modulation instability, colliders

PACS: $41.75 . \mathrm{Lx}, 52.40 . \mathrm{Mj}, 29.27 . \mathrm{Bd}$

\section{INTRODUCTION}

Next generation lepton colliders should reach TeV scale energy. Circular colliders are not feasible at these energies due to synchrotron radiation losses. Hence, future collider designs are based on linear colliders. Currently, metallic cavities can achieve a maximum accelerating gradient around $100 \mathrm{MV} / \mathrm{m}$. Thus, to reach $\mathrm{TeV}$ energy in a linear accelerator, the length of the machine will be about tens of kilometers. Plasmas, a medium of ions and free electrons, can support very large electric field and therefore could be used to accelerate particles to relativistic energies in a short distance. Recently, both laser-driven and electron-driven plasma-wakefield acceleration were demonstrated. But, they have been limited to distances of few meters [1][2]. To accelerate an electron bunch to $\mathrm{TeV}$ using these schemes, many stages will have to be combined. Such multi-stage design may be challenging to implement. Proton-driven plasma-wakefield acceleration promises to accelerate electrons to $\mathrm{TeV}$ in a single stage.

High energy protons are readily available from conventional accelerators and hence it is possible to accelerate electron bunches in the wake of the proton bunch up to several $\mathrm{TeV}$ [3]. The maximum energy gain of electrons accelerated in a single plasma wake is limited to roughly twice the energy of the particles in the driving bunch. Given that protons can be accelerated to $\mathrm{TeV}$ energies in conventional accelerators, it should be possible to accelerate electron bunches in the wake of a proton driving-bunch to energies up to the $\mathrm{TeV}$ regime in one pass through the plasma.The goal of this project, named PROTOPLASMA, is to experimentally demonstrate proton-driven plasmawakefield acceleration using the proton beam from the Main Injector $(120 \mathrm{GeV})$ to accelerate an electron beam (5 $\mathrm{MeV}$ ) up to $1 \mathrm{GeV}$ in 2 meters in the Tevatron beamline at Fermilab. In this paper, we summarize our first round of design parameters and simulation for this experiment using the Tevatron beamline.

\section{TECHNICAL REQUIREMENTS}

In this section, we discuss the parameter set of the existing proton beam at Fermilab and show that acceleration of electron beam could be possible with a proton-driven plasma-wakefield acceleration scheme. The key parameters required to demonstrate proton beam driven plasma-wakefield acceleration are the proton beam energy, intensity, bunch length, momentum spread and transverse proton beam size. We plan to inject the proton beam from the main injector $(120 \mathrm{GeV})$. The number of protons in the drive bunch is $10^{11}$. The beam has a momentum spread of $10^{-4}$. The normalized beam emittance is $20 \pi \mathrm{mm}$-mrad while the bunch length can be varied between $1 \mathrm{~ns}-500 \mathrm{ps}$. The 
beam will be focused to a spot size of $50 \mu \mathrm{m}$. The proton beam will be injected into the Tevatron beam line at F0. The Tevatron sector beam line will then be used to transport the beam up to B0 and into the plasma cell that will generate wakefields through self-modulation. At this stage, an electron beam $(5 \mathrm{MeV})$ will be injected into the plasma cell at the right phase to be accelerated by the wakefield. The proton beam will then be directed to the beam dump while the spectrometer magnet will deflect the electron beam into an electron detector. Other diagnostics like OTR/CTR will also be installed to give information about the spatial and temporal development of the proton bunch current profile during the modulation process. A streak camera will be used to resolve structures $<1 \mathrm{ps}$. A schematic of the experiments is shown in Fig. 1

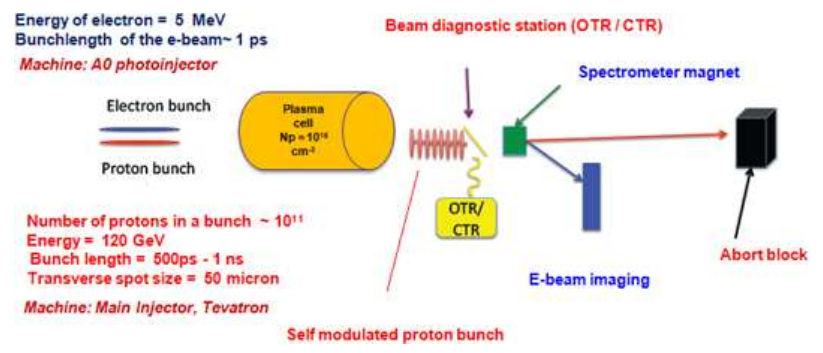

FIGURE 1. Schematic of the PROTOPLASMA experiment. The first phase of the project is planned to observe the selfmodulation of the proton bunch. In the next phase, an electron beam will be injected for plasma-wakefield acceleration experiments.

\section{Generating short proton bunches}

While proton beams from the Main Injector has the required energy, the bunch length of proton beam is in 10 s of centimeters NOT millimeters. Most of the existing proton accelerators produce bunches that are around two orders of magnitude longer than the plasma period that is required to drive a plasma wakefield. The main reason is the beam current (bunch charge over the bunch length) is directly related to transverse instabilities and hence by using a long bunch the beam current can stay below the threshold of those instabilities. Other reason is to minimize the energy spread. This is done by rotating the longitudinal phase space, which invariably increases the bunch length.

One obvious way to obtain short bunches would be to compress the proton bunch. But, the RF power needed will be prohibitive: the equilibrium bunch length in a proton storage ring scales inversely to the fourth root of the RF voltage. To compress a bunch from tens of centimeters to sub-mm will required formidable RF power. In other words, though current technology does allow one to generate or compress and obtain millimeter scale proton bunches, it is a very expensive option.

A proton driver must be short to excite the plasma wake resonantly. Studies on the interaction between a long proton bunch and plasma have shown that a strong density modulation occurs at the order of half the plasma wavelength, which excites the plasma wake resonantly to accelerate the externally injected electrons through self-modulation[4]. For a plasma cell of density $10^{15} \mathrm{~cm}^{-3}$, the plasma wavelength will be $1 \mathrm{~mm}$, so a gradient of $1 \mathrm{GeV} / \mathrm{m}$ should be possible with the Fermilab beam parameters. This is an upper-limit and does not include other effects that might limit the acceleration gradient such as phase slippage.

\section{Measuring the energy gain}

In the initial phase of the experiment, there will be no electron beam and we plan to measure the gradient by measuring the energy loss of the proton beam using a dispersive lattice. So, a key diagnostic in PROTOPLASMA experiment is the spectrometer beamline that can resolve the energy loss of the proton beam after the plasma cell. The requirement is to measure $1 \mathrm{GeV}$ energy loss over $120 \mathrm{GeV}$. i.e. $\leq 0.8 \%$ In this section, we describe the beamline optics that will allow us to measure such low energy spread beam with enough resolution. The beamline from F0 to A0 is one of the sectors (FODO arcs) of the Tevatron and thus gives a good control over dispersion. In order to resolve the energy spread $\delta>>\frac{\sqrt{\beta_{x} \varepsilon}}{\eta}$. In our case, the $\beta_{x}=30 \mathrm{~m}$ and $\eta=4.4$ and thus giving a resolution of $\sim 0.2 \%-$ four times smaller than the requirement. Thus, the Tevatron beamline is ideally suited to measure this energy loss. 
There are also other added benefits of using the Tevatron beamline. The low-beta quads that were used in the Tevatron for Collider Detector Facility (CDF) [5] will be powered thus giving us a precise control of the beam spot size entering the plasma cell. Other issues that has to be worked out include radiation loss, seeding the plasma for reliable self-modulation, plasma cell design and chromatic aberration effects on the low-beta quads.

\section{LAYOUT OF THE PROTOPLASMA EXPERIMENT}

The PROTOPLASMA experiment will be run parasitically with the proposed ORKA experiment. The ORKA [6] will make a precise measurement of $\left(K^{+} \rightarrow \pi^{+} v \bar{v}\right)$ decay. If the decay occurs at the standard model rate, 200 events are expected per year of operation. The experiment will use the CDF magnet with a new detector inside the solenoid and will study the decays of the stopped kaons. The primary beam will be $95 \mathrm{GeV}$ protons from the MI in a $20 \mu$ s slow spill. The primary beamline will be for the most part be a reconstruction of the A sector of the Fermilab Main Ring. The production target will be placed near the entrance to the collision hall. The CDF central detector will be moved off-axis, and the secondary beamline will have a dog-leg configuration. To achieve a $5 \mathrm{~m}$ offset between the beam axis and the detector axis, all of the CDF external muon components will be dismantled, the central detector will be moved $3 \mathrm{~m}$ north (outside the ring), and the primary beamline will enter the hall $2 \mathrm{~m}$ south of the Tevatron beam axis. In this configuration, there is sufficient space for the primary beam freely to pass south of the CDF magnet for the PROTOPLASMA experiment. The drawing of the CDF hall is shown in Fig. 2

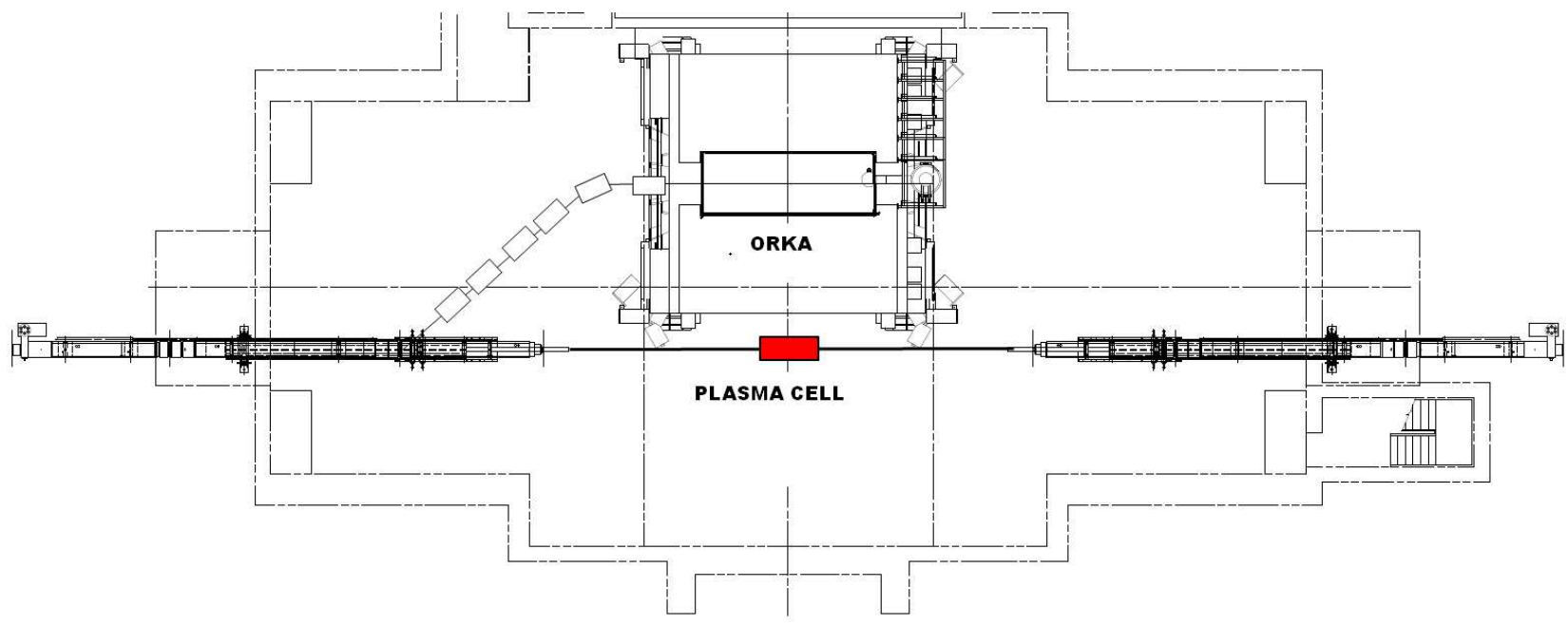

FIGURE 2. The floor plan of the Collider Detector Facility (CDF) showing the layout of the PROTOPLASMA experiment. The experiment will also be synergistic with the ORKA high energy experiment planned at CDF. A dogleg will inject the proton bunch into the ORKA detector.

\section{PARTICLE TRANSPORT USING SYNERGIA}

Synergia 2.1 is a parallel code composed of several modules, such as linear/non-linear optics, space charge, and impedance, etc [7] for particle beam simulation and transport. Using Synergia, we performed the multi-particle beamline simulation of the self-modulated proton bunch after the plasma cell through the low-beta quads in the straight section in the Tevatron beamline. Synergia 2.1 is also capable of optimizing the beamline optics in conjunction with the nonlinear optimization library, NLopt. Three quadrupoles, which are at the downstream of the plasma cell, were chosen to minimize the betatron functions on the screen. With the local derivative-free algorithm of the NLopt library, we found the optimal strengths of quadrupoles. With the particles of either QuickPIC or OSIRIS results, Synergia 2.1 simulated the beam dynamics through the optimized beamline. The dispersion function and the betatron function are shown in Fig. 3 respectively. 

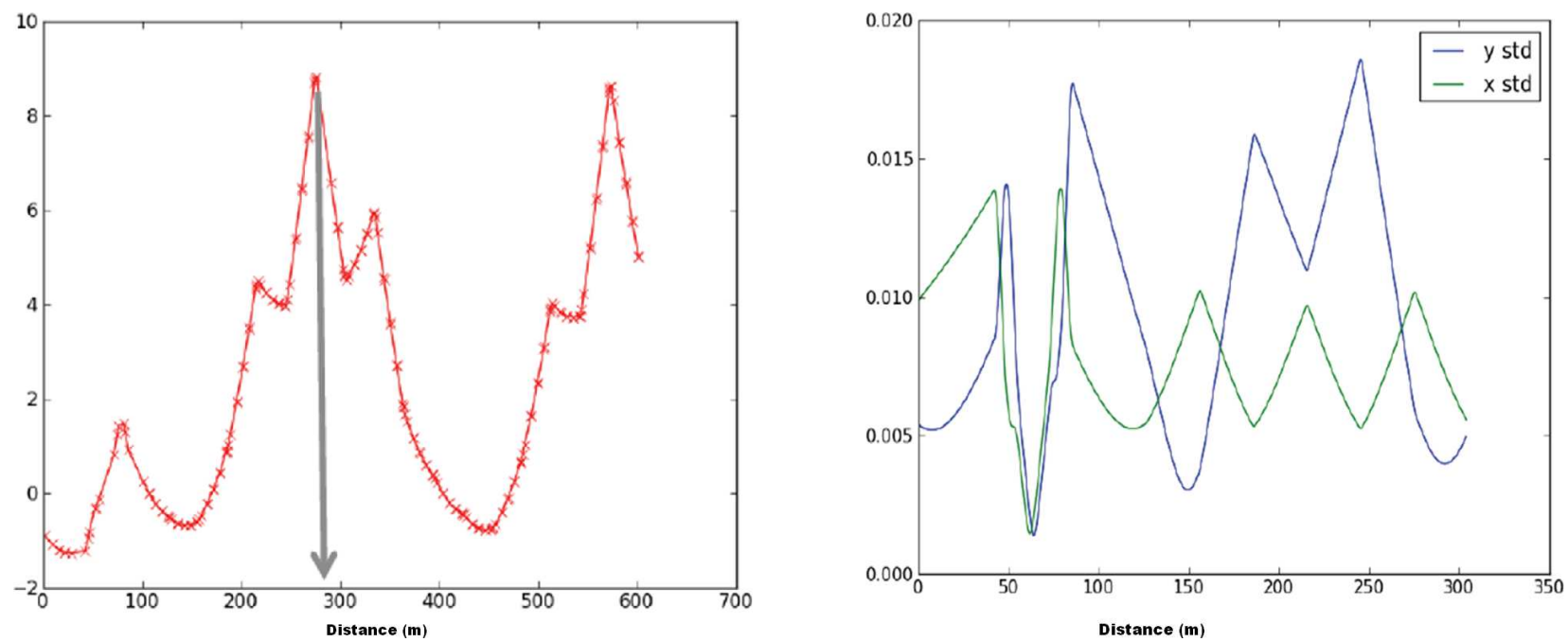

FIGURE 3. The dispersion function (left) and the beta function (right) as a function of position along the Tevatron beamline. A screen will be installed at the maximum dispersion position to observe the energy loss with the plasma cell on/off

\section{PARTICLE SIMULATION USING OSIRIS AND QUICKPIC}

Osiris [8] and QuickPIC [9] was used to simulate the self-modulation expected for the PROTOPLASMA parameters. We also updated the codes to enable the beam initialization with Twiss parameters (which describes the transverse phase space distribution of the beam). This is important for the simulation because the bunch length is comparable with the beam $\beta^{*}$. The Fig. 4 shown below is the simulation result from Osiris with 2D cylindrical coordinates showing a snapshot of the bunch charge density at the time when the beam center is propagating inside the plasma after a distance of $62 \mathrm{~cm}$. The proton beam already has a significant self modulation, resulting in the micro bunching in the middle and rear part of the beam. The distance between each two micro bunches is equal to the plasma wavelength. Fig. 5 shows the energy modulation on the proton beam at that time. The energy modulation also happens in the middle and rear part of the beam. The maximum energy change at this time is around $200 \mathrm{MeV}$. Detailed description of the simulation with the Fermilab parameters is discussed in [10].

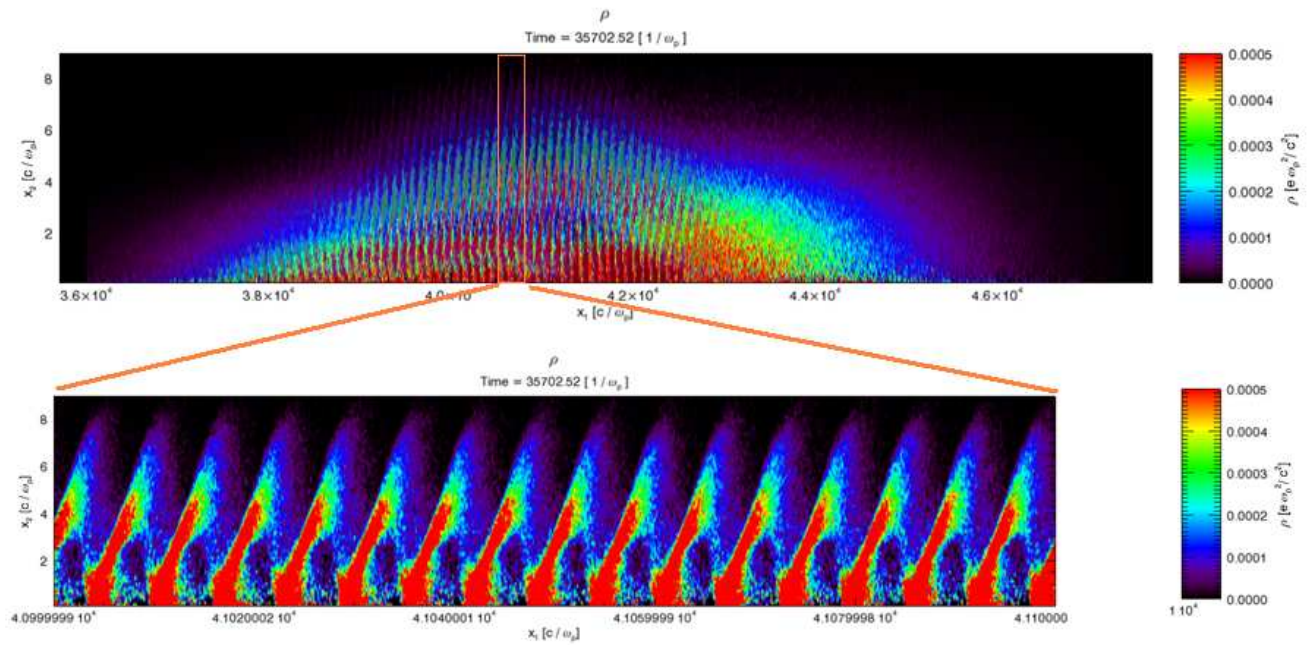

FIGURE 4. Snapshot of a self modulated proton beam at the time when the beam center propagating in the plasma for $62 \mathrm{~cm}$. The total beam particle number is $\mathrm{N}=1 \times 10^{11}$; the initial beam focus spot size (r.m.s. value) is $\sigma_{r}=100 \mu \mathrm{m}$; the initial beam length (r.m.s. value) is $\sigma_{z}=10 \mathrm{~cm}$; the initial beam emittance is $\varepsilon_{n}=3.33 \mathrm{~mm}$ mrad; the beam is focused at $60 \mathrm{~cm}$ inside the plasma. The initial plasma density is $1 \times 10^{16} \mathrm{~cm}^{-3}$. (The beam density is normalized to the initial plasma density.) 


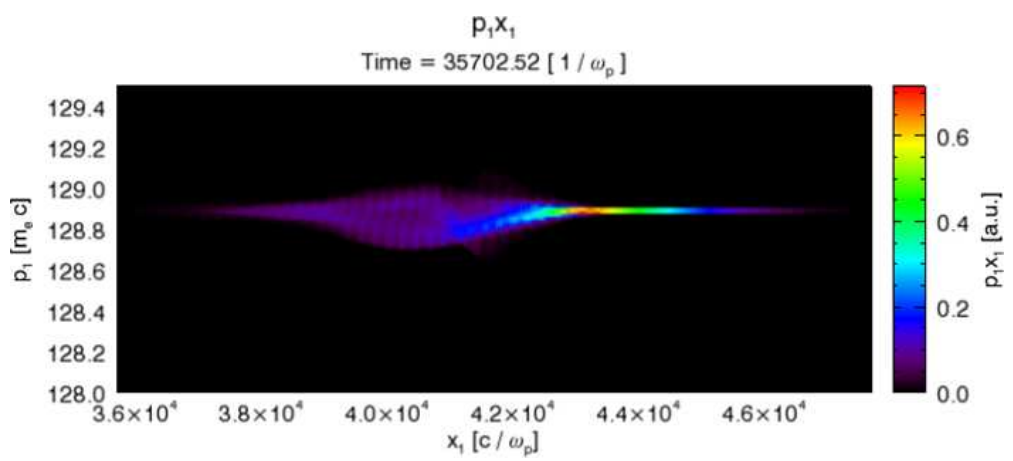

FIGURE 5. The proton beam $\mathrm{p} 1-\mathrm{x} 1$ phase space distribution at the time when the beam center propagating in the plasma for 62 $\mathrm{cm}$.

\section{PLASMA CELL}

A plasma cell will be commissioned with the necessary diagnostics and good control over plasma density. In order to produce homogenous plasma and reduce cost and time in designing, we plan to use plasma sources similar to the SLAC E-157 experiments as shown in Fig. 6. These plasma sources typically use pre-ionized Li vapor. A neural Lithium vapor density up to $10^{16} \mathrm{~cm}-3$ can be generated. The heat pipe oven provides a homogeneous metal vapor over a well defined length. The vapor evaporates from the melted metal uniformly distributed by capillary action along a wick lining the pipe interior. The hot Lithium vapor is confined by Helium gas window. Further options on the plasma cell design are currently under research.

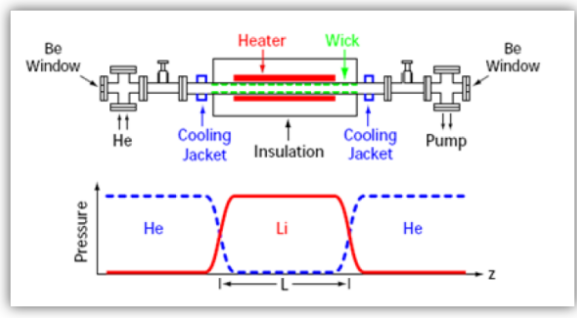

FIGURE 6. A schematic of the plasma cell used in the SLAC experiment. The Helium gas is used as window to confine the Li vapor to the central part of the oven. Li vapor density can vary from $10^{14}$ to $10^{16} \mathrm{~cm}^{-3}$ by heating the $\mathrm{Li}$.

\section{SUMMARY}

In this work, we have described the planned proton-driven wakefield acceleration at Fermilab named PROTOPLASMA. We have identifed the Tevatron beamline being ideally suited for this experiment because of the availability of the spectrometer with adequate resolution and the superconducting low-beta quadrupoles for spot size control. Extensive particle simulations also predict the possibility of observing self-modulation at $120 \mathrm{GeV}$. The results of this project may provide insight and may also lead to added option for the future design of lepton and muon colliders.

\section{ACKNOWLEDGMENTS}

We thank the Community Petascale Project for Accelerator Science and Simulation (COMPASS) collaboration for providing the computational resources for this project. One of the authors (J. T.) would like to thank V. Shiltsev, D.Still and G. Annala for their assistance with the Tevatron beamline questions. 


\section{REFERENCES}

1. I. Blumenfeld, C. Clayton, F. Decker, M. Hogan, C. Huang, R. Ischebeck, R. Iverson, C. Joshi, T. Katsouleas, N. Kirby, et al., Nature 445, 741-744 (2007).

2. W. Leemans, B. Nagler, A. Gonsalves, C. Toth, K. Nakamura, C. Geddes, E. Esarey, C. Schroeder, and S. Hooker, Nature physics 2, 696-699 (2006).

3. A. Caldwell, K. Lotov, A. Pukhov, and F. Simon, Nature Physics 5, 363-367 (2009).

4. N. Kumar, A. Pukhov, and K. Lotov, Physical review letters 104, 255003 (2010).

5. F. Abe, D. Amidei, G. Apollinari, G. Ascoli, M. Atac, P. Auchincloss, A. Baden, A. Barbaro-Galtieri, V. Barnes, E. Barsotti, et al., Nuclear Instruments and Methods in Physics Research Section A: Accelerators, Spectrometers, Detectors and Associated Equipment 271, 387-403 (1988).

6. J. Comfort, D. Bryman, L. Doria, T. Numao, A. Sher, D. Vavilov, D. Jaffe, S. Kettell, L. Littenberg, E. Worcester, et al., beamdocs.fnal.gov (2011).

7. J. Amundson, P. Spentzouris, J. Qiang, and R. Ryne, Journal of Computational Physics 211, 229-248 (2006).

8. R. Fonseca, L. Silva, F. Tsung, V. Decyk, W. Lu, C. Ren, W. Mori, S. Deng, S. Lee, T. Katsouleas, et al., Computational Science ICCS 2002 pp. 342-351 (2002).

9. C. Huang, V. Decyk, C. Ren, M. Zhou, W. Lu, W. Mori, J. Cooley, T. Antonsen, and T. Katsouleas, Journal of Computational Physics 217, 658-679 (2006).

10. W. An, et al., in the proceedings of Advanced Accelerator workshop (2012). 